\title{
Histopathological analysis of endometrial biopsies in dysfunctional uterine bleeding
}

\author{
Nepal N ${ }^{1}$, Choudhary PK ${ }^{1}$, Mainali $\mathrm{N}^{1}$ \\ ${ }^{\text {I} D e p a r t m e n t ~ o f ~ P a t h o l o g y, ~ N o b e l ~ M e d i c a l ~ C o l l e g e ~ T e a c h i n g ~ H o s p i t a l, ~ B i r a t n a g a r, ~ N e p a l ~}$
}

\section{Keywords: \\ Dilatation and Curettage; Dysfunctional uterine bleeding: \\ Histopathology.}

\begin{abstract}
Background: It is estimated that $9-30 \%$ of women of reproductive age suffer from menorrhagia. Because most cases are associated with anovulatory menstrual cycles, adolescent and perimenopausal women are particularly vulnerable to this particular condition. The aim of this study was to evaluate the histopathological pattern of endometrial biopsy from patients presenting with dysfunctional uterine bleeding.

Materials and Methods: This is a cross-sectional observational study done in Nobel Medical College, Biratnagar, Nepal for a period of three years from June 2012 to June 2015. All the patients presenting with dysfunctional uterine bleeding and who underwent endometrial biopsy were included in this study. Distribution of various histopathological patterns was observed in the different age groups.

Results: A total of 300 cases were included in my study. The commonest pattern in these patients was proliferative endometrium 61 (\%). The commonest pathology was simple cystic hyperplasia (13.3\%). Other patterns identified were secretory endometrium, chronic endometritis and pill endometrium. Malignant lesion comprised of $8(2.7 \%)$ of patients and the majority was in $>50$ years followed by in between 41-50 years of age. Atrophic endometrium was the commonest finding in post menopausal bleeding, comprising of 11 cases (3.6\%), followed by endometrial carcinoma of 8 cases $(2.7 \%)$.

Conclusion: As observed from the study, there is an age specific association of endometrial bleeding, with highest incidence in perimenopausal age group. Hence, dilatation and curettage is helpful for diagnosis, to assess therapeutic response and to know the pathological incidence of organic lesions in cases of dysfunctional uterine bleeding. female population.
\end{abstract}

\section{INTRODUCTION}

Dysfunctional uterine bleeding is one of the commonest problems presented in the developing countries. As women suffer from many gynaecological diseases, dysfunctional uterine bleeding is one of them, which has significant morbidity in that it interferes with their personal, family

\section{Correspondence:}

Dr. Niraj Nepal, MBBS, $M D$

Department of Pathology,

Nobel Medical College Teaching Hospital

Email:nepalniraj@gmail.com and social life. This is one of the commonest presenting conditions of the female seeking gynaecological advice and treatment. It is estimated that $9-30 \%$ of women of reproductive age suffer from menorrhagia. The prevalence increases with age, peaking just before menopause. Because most cases are associated with anovulatory menstrual cycles, adolescent and perimenopausal women are particularly vulnerable to this particular condition. ${ }^{1}$ 
Table: 1 Microscopic patterns of endometrial biopsy in different age groups.

\begin{tabular}{|c|c|c|c|c|c|}
\hline \multicolumn{6}{|c|}{ Age Group } \\
\hline Endometrial pattern & 21-30 Yrs & 31-40 Yrs & 41-50 Yrs & $>50 \mathrm{Yrs}$ & Total \\
\hline Simple cystic hyperplasia & 6 & 12 & 20 & 2 & 40 \\
\hline Complex hyperplasia & 0 & 3 & 2 & 5 & 10 \\
\hline Adenomatous hyperplasia & 4 & 10 & 17 & 5 & 36 \\
\hline Proliferative endometrium & 13 & 20 & 28 & 0 & 61 \\
\hline Proliferative endometrium with focal hyperplasia & 0 & 4 & 5 & 1 & 10 \\
\hline Secretory endometrium & 9 & 16 & 10 & 0 & 35 \\
\hline Pill endometrium & 3 & 15 & 12 & 0 & 30 \\
\hline Chronic endometritis & 3 & 9 & 12 & 2 & 26 \\
\hline Endometrial carcinoma & 0 & 0 & 3 & 5 & 8 \\
\hline Arias stella reaction & 0 & 3 & 0 & 0 & 3 \\
\hline Irregular shedding & 0 & 2 & 2 & 0 & 4 \\
\hline Atrophic endometrium & 0 & 0 & 4 & 7 & 11 \\
\hline Inadequate & 0 & 3 & 5 & 3 & 11 \\
\hline Blood clots only & 0 & 8 & 2 & 5 & 15 \\
\hline Total & 38 & 105 & 122 & 35 & 300 \\
\hline
\end{tabular}

Dysfunctional uterine bleeding (DUB) is abnormal uterine bleeding in a premenopausal woman resulting from alterations in the normal cyclical changes of the endometrium and without an underlying specific pathological cause such as endometritis, polyps, exogenous hormones, hyperplasia, or carcinoma. ${ }^{1}$ Bleeding not associated with an organic cause in women of child-bearing age belongs to the large and somewhat nebulous category known as dysfunctional uterine bleeding. Examination of specimens obtained on $\mathrm{D} \& \mathrm{C}$ or endometrial biopsy is a continuous source of frustration for the pathologist. Sometimes, provided with minimal clinical information or material taken at an inappropriate moment in the menstrual cycle, one is unable to recognize any abnormality. At most, changes can be detected that only confirm what the gynecologist already knows - i.e., that the patient has an abnormal bleeding ${ }^{2}$.

The endometrial biopsy by dilatation and curettage is chosen to evaluate dysfunctional uterine bleeding because it has several advantages over other diagnostic methods. The hormonal assay is very expensive and laboratories with hormonal assay are not available in rural area.

\section{MATERIALS AND METHODS}

This study was done in the department of pathology of Nobel medical college over a period of three years from June 2012 to June 2015. Prior to the study, permission from institutional review committee was obtained. Endometrial sampling tissue obtained by Dilatation and Curettage from the department of Gynaecology of patients presenting with Dysfunctional Uterine Bleeding. Inclusion criteria: Endometrial tissue from patients of all age groups clinically diagnosed as DUB was included in the study. Exclusion criteria: 1) Patients presenting with DUB due to pregnancy related complications 2) Organic lesions involving the genital tract and organs like leiomyomas and adenomyosis, genital tract infections, systemic causes and other lesions 3) Hysterectomy specimens. The endometrial samples received was fixed in 10\% formalin for 12-24 hours and the tissue was taken for routine processing as per guidelines and $0.5 \mu \mathrm{m}$ of thickness sections were made from the paraffin blocks were stained with Haematoxylin and Eosin (H \& E) and examined under the microscopy. Relevant clinical data was collected from the hospital and laboratory records when required. Microscopic examination was done by two expert pathologists, individually to reduce observer bias.

\section{RESULTS}

A total of 300 samples of endometrial biopsy obtained from the gynaecological department were included in the study. All the endometrial biopsies obtained for the study were obtained by dilatation and curettage (D \& C) method. Patient's age ranged from 21- 65 years. The maximum number of patients was in the group between 41-50 years of age, total of $122(40.6 \%)$. Highest incidence of DUB was found in 41-50 years of age group. The commonest pattern in 41-50 years patients was proliferative endometrium. Out of 300 patients, $223(74.33 \%)$ were premenopausal whereas $77(25.66 \%)$ were postmenopausal. Proliferative endometrium was the commonest and predominant findings irrespective of all groups $(20.3 \%)$, followed by simple cystic hyperplasia (13.3\%). (11.6\%) of endometrial biopsies were diagnosed as secretory phase endometrium. Endometrial hyperplasia was the most common pathology encountered. $12 \%$ biopsies showed features of Adenomatous hyperplasia and $3.3 \%$ biopsies showed features of complex atypical 
hyperplasia.

Incidence of simple cystic hyperplasia was highest in the 4th decade of life suggesting that the incidence of endometrial hyperplasia increases with age. Of the 300 endometrium samples $10 \%$ samples showed features of pill endometrium, which could be due to hormone effect and majority of the patients presented with menorrhagia. Three (1\%) patients were diagnosed as Arias Stella reaction. Twenty six (8.6\%) of patients were diagnosed having chronic endometritis. Malignant lesion comprised of $8(2.7 \%)$ of patients. Majority was in $>50$ years followed by in between $41-50$ years of age as demonstrated in table 1 .

\section{DISCUSSION}

Dysfunctional uterine bleeding is abnormal uterine bleeding in a premenopausal woman resulting from alterations in the normal cyclical changes of the endometrium and without an underlying specific pathological cause such as endometritis, polyps, exogenous hormones, hyperplasia, or carcinoma. It is considered to be the diagnosis of exclusion made when there is no identifiable medical cause. Abnormal and excessive endometrial bleeding without structural pathology occurs in reproductive women of all ages but is more common in adolescent and perimenopausal women. ${ }^{3}$ The causes for abnormal uterine bleeding can be divided as follows: reproductive disease, iatrogenic cause and associated with systemic disease. So, when no organic cause is identified, then a diagnosis of DUB can be made or assumed. In about $25 \%$ of the patients, the abnormal uterine bleeding is the result of a well-defined organic abnormality. ${ }^{4}$ As the women get older the incidence of structural abnormality including malignancy increases. ${ }^{3,5}$ Routine tests for DUB includes complete blood count, prothrombin time , Activated partial thromboplastin time and liver function test to rule out any coagulation and bleeding disorders. Regarding women in reproductive age group, serum level and urine level of human chorionic gonadotropin levels are measured to rule out pregnancy and other associated conditions. To rule out endocrine causes of dysfunctional uterine bleeding includes, thyroid function tests, follicle stimulating hormones, luteinizing hormone and prolactin levels are assessed in the patients. All adolescents with menorrhagia should undergo evaluation for coagulopathy. ${ }^{6}$ When none of these causes are identifiable, imaging studies are done which includes pelvic ultrasound (USG) and transvaginal USG and tissue sampling. So the choice of investigation for the gynaecologists is dilatation and curettage, which is considered to be a diagnostic as well as therapeutic procedure. Organic diseases of the genital tract may be missed even by curettage. The sensitivity in detecting intrauterine pathology by curettage is $94-97.5 \% .^{7}$ The commonest age group presenting with excessive bleeding in my study was 41-50 years. A similar incidence was reported by Yusuf et al. and Muzaffar et al. ${ }^{6,8}$ in their study of endometrium. In this study, as others studies revealed that proliferative lesions like disordered proliferative pattern, endometrial hyperplasia and secretory endometrium occur more commonly in the age group 41-50 years. The reason for increased incidence of dysfunctional uterine bleeding in this age group (41-50 years) may be due to the fact that these patients are in their climacteric period. Due to shortening of the menstrual cycle and often becoming intermittently anovulatory, ovarian follicles decrease in number so as the estradiol level, menopausal women are prone to suffer from this condition. High proportion of the cases in studied revealed normal physiological phases like proliferative, secretory and atrophic endometrium. Bleeding in the proliferative phase is due to anovulatory cycles and in secretory phase is due to ovulatory dysfunctional uterine bleeding. Large number of cases revealed disordered proliferative pattern in my study. Disordered proliferative phase is considered to be one of the proliferative lesions in the endometrium, which includes carcinoma on one side and intervening stages of hyperplasia on the other side. It has been used in different ways as mentioned above and is therefore difficult to define. It denotes an endometrial appearance that is hyperplastic but without an increase in endometrial volume. ${ }^{9}$ It is also defined as proliferative phase endometrium which does not seem appropriate in the menstrual cycle, but is abnormal enough to be considered hyperplastic. The histological feature of disordered proliferative phase resembles features of simple hyperplasia, but the process is more suggestive of focal than diffuse. The exact mechanism of bleeding in the patients more than 50 years cannot be established, but it can be due to various anatomic vascular variations or due to local abnormal hemostatic mechanism. Due to the presence of thin walled veins, superficial to the expanding cystic glands makes the vessels more vulnerable to injury. High risk factors such as obesity, diabetes, increased intake of animal fat and especially sedentary life styles increases the incidence of endometrial hyperplasia. Endometrial hyperplasia could be a precursor of fertile soil for conversion into endometrial carcinoma that is why it is important to be identified by the pathologists. As observed in my study, the incidence of endometrial carcinoma had more prevalence in the age group ranging from 41-60 years of age. In various studies, malignancy of the genital tract accounted for $30-50 \%$ of cases of bleeding in the postmenopausal period. ${ }^{10}$ Regarding the cases of endometritis observed in 26 patients, which was quite more in number, this has to be taken in consideration because with the specific treatment, endometrium starts functioning normally. ${ }^{11}$

\section{CONCLUSION}

Dilatation and curettage is an important tool or procedure to establish or reveal the diagnosis for different patterns of dysfunctional uterine bleeding, comprising of normal proliferative, secretory phase to irregular shedding, pill endometrium and cystoglandular hyperplasia. It can also be used to exclude organic pathology which mimics dysfunctional uterine bleeding like endometrial polyp, 
chronic endometritis or endometrial carcinoma etc. Based on the above sayings, endometrial biopsies is very useful and is an important diagnostic tool to distinguish between anovulatory from ovulatory DUB. It is also useful for the diagnosis of endometrial hyperplasia and endometrial carcinoma. Concluding, dilatation and curettage is an important tool used for the diagnosis, to plan the management of the patients, to access the treatment response and to exclude the organic lesions prior to the surgery in patients presenting with dysfunctional uterine bleeding.

\section{REFERENCES}

1. Robert J Kurman. Blaustein's Pathology of the Female Genital Tract. Springer. 5th Edition.2004.1391p.

2. Juan Rosai. Rosai and Ackerman's Surgical Pathology. Mosby. 9th Edition. 2005.2892p.

3. Bayer SR, DeCherney AH. Clinical Manifestations and Treatment of Dysfunctional Uterine Bleeding. JAMA. 1993;269:1823-8. Crossref

4. Chabra S, Jaswal M, Nangia V. Uterine size, Endometrium Fertility in women with dysfunctional uterine haemorrhage. J Obstet Gynaecol $1992 ; 42: 692-4$
5. Sharma JB. Dysfunctional Uterine Bleeding (DUB). Obstetrics and Gynaecology Today, 2000;5: 20-5.

6. Muzaffar M, Akhtar KA, Yasmin S, Mahmood-Ur-Rehman, Iqbal W, Khan MA. Menstrual irregularities with excessive blood loss: a clinico-pathological correlation. J Pak Med Assoc 2005;55: 486-9. Crossref

7. Quinn MA, Anderson MC, Coulter CAE, Soutter WP Malignant Disease of the uterus In: Shaw RW, Soutter WP, Stanton SL, eds. Gynaecology. London, Churchill Livingston, 1997.pp585-603.

8. Yusuf NW, Nadeem R, Yusuf AW. Dysfunctional Uterine Bleeding: A Retrospective Clinicomorphological Study over Two Years. Pak J Obstet Gynaecol 1996;9:27-30.

9. Silverberg SG. Problems in the differential diagnosis of endometrial hyperplasia and carcinoma. Mod Pathol 2000;13:309-27. Crossref

10. Anonymous. Carcinoma of the endometrium. In: Padubidri VG, Daftary SN, eds. Shaw's textbook of Gyaenocology, 12th ed., New Delhi, Churchill Livingston, 1999. pp 302-9.

11. George AV, Guylaine L, Graves GR. Guidelines for the management of abnormal uterine bleeding. J Obstet Gynaecol Can 2001;23:704-9. 